\title{
THE BIODIVERSITY OF CATALYTIC SUPER-BROWNIAN MOTION
}

\author{
By Klaus FleischmanN ${ }^{1}$ AND Achim KLenKe ${ }^{2}$ \\ Weierstrass-Institut für Angewandte und Stochastik and \\ Universität Erlangen-Nürnberg
}

\begin{abstract}
In this paper we investigate the structure of the equilibrium state of three-dimensional catalytic super-Brownian motion where the catalyst is itself a classical super-Brownian motion. We show that the reactant has an infinite local biodiversity or genetic abundance. This contrasts to the finite local biodiversity of the equilibrium of classical super-Brownian motion.

Another question we address is that of extinction of the reactant in finite time or in the long-time limit in dimensions $d=2,3$. Here we assume that the catalyst starts in the Lebesgue measure and the reactant starts in a finite measure. We show that there is extinction in the long-time limit if $d=2$ or 3 . There is, however, no finite time extinction if $d=3$ (for $d=2$, this problem is left open). This complements a result of Dawson and Fleischmann for $d=1$ and again contrasts the behaviour of classical super-Brownian motion.

As a key tool for both problems, we show that in $d=3$ the reactant matter propagates everywhere in space immediately.
\end{abstract}

1. Introduction and results. Catalytic super-Brownian motion (CSBM) $X^{\varrho}$ is the measure-valued (finite variance) branching diffusion on $\mathbb{R}^{d}$ where the local branching rate is given by a space-time varying medium $\varrho$, the so-called catalyst. For a survey on CSBM and a variety of different spatial branching models, see Klenke [14]. The case on which we focus here is where $\varrho$ is a random sample of classical super-Brownian motion (SBM). So that the reactant is nondegenerate, we have to restrict to $d \leq 3$.

This model was constructed in Dawson and Fleischmann [1] and has been considered under various aspects, for instance, also in [2] and [11]. This paper is meant to be concise, not self-contained, so we skip the usual heuristics and repetitive constructions and refer only to the above-mentioned papers.

1.1. Biodiversity. The main subject of this paper is the local biodiversity or genetic abundance of the equilibrium states in $d=3$. The investigation of biodiversity is a booming field in biology. Roughly speaking, biodiversity is a measure for the number of species per square meter in an ecosystem. Our ecosystem is the reactant of three-dimensional catalytic super-Brownian

Received July 1999; revised January 2000.

${ }^{1}$ Partly supported by the Deutsche Forschungsgemeinschaft.

${ }^{2}$ Partly supported by the Deutsche Forschungsgemeinschaft and Don Dawson's Max Planck Grant for international collaboration.

AMS 1991 subject classifications. Primary 60J80; secondary 60G57, 60K35.

Key words and phrases. Superprocess, genetic abundance, equilibrium states, extinction, instantaneous propagation of matter. 
motion in a steady state. Before we make mathematical statements about its biodiversity, we have to fix this notion.

It is well known that (if $d \geq 3$ ) SBM has a unique ergodic equilibrium with intensity $i_{c}\left(i_{c}>0\right)$. We denote by $\mathbb{P}_{-\infty, i_{c} l}$ the law of the corresponding equilibrium process $\left(\varrho_{t}\right)_{t \in \mathbb{R}}$. For fixed $\varrho$ consider $X^{\varrho}$ started at time $t$ in $i_{r} l, i_{r}>0$, ( $l$ is the Lebesgue measure), and denote its law by $P_{t, i_{r} l}^{\varrho}$. Letting $t \rightarrow-\infty$, we obtain $P_{-\infty, i_{r} l}^{\varrho}$ and $\left(\varrho_{t}, X_{t}^{\varrho}\right)_{t \in \mathbb{R}}$ is the (bivariate) equilibrium process (see [2])

$$
\mathbb{P}_{-\infty, i_{c} l}\left[P_{-\infty, i_{r} l}^{\varrho}\left[\left(\varrho_{T+t}, X_{T+t}^{\varrho}\right)_{t \in \mathbb{R}} \in \bullet\right] \in \bullet\right] \text { is independent of } T \in \mathbb{R} .
$$

Furthermore $\mathbb{P}_{-\infty, i_{c} l}$ almost surely $E_{-\infty, i_{r} l}^{\varrho}\left[X_{0}^{\varrho}\right]=i_{r} l$, and $P_{-\infty, i_{r} l}^{\varrho}\left[X_{0}^{\varrho} \in \bullet\right]$ is infinitely divisible.

Note that the outlined $\mathbb{P}$ refers to the medium and the italic $P$ refers to the reactant. All other quantities' laws are denoted by a bold P. Expectations are denoted by the symbol $E$ in respective fonts.

We are interested in the number of families that contribute to $X_{0}^{\varrho}(B)$ for, say, the unit ball $B$. To make this notion precise, recall that an infinitely divisible random measure $Y$ [with values in $\mathscr{M}\left(\mathbb{R}^{d}\right)$, the space of Radon measures on $\left.\mathbb{R}^{d}\right]$ has a cluster representation

$$
Y=\alpha+\sum_{i} \chi_{i}
$$

where $\alpha \in \mathscr{M}\left(\mathbb{R}^{d}\right)$ is the deterministic component of $Y$ (or the essential infimum of $Y)$. The $\chi_{i} \in \mathscr{M}\left(\mathbb{R}^{d}\right)$ are the "points" of a Poissonian point process on $\mathscr{M}\left(\mathbb{R}^{d}\right) \backslash\{0\}$ with intensity measure $\mathbf{Q}$, which is called the canonical measure of $Y$. We can reformulate (1.2) as the classical Lévy-Hincin formula for the Laplace transforms

$$
-\log \mathbf{E}\left[e^{-\langle Y, \varphi\rangle}\right]=\langle\alpha, \varphi\rangle+\int_{\mathscr{M}\left(\mathbb{R}^{d}\right)} \mathbf{Q}(d \chi)\left(1-e^{-\langle\chi, \varphi\rangle}\right) .
$$

Here $\varphi \in C_{c}^{+}\left(\mathbb{R}^{d}\right)$ (the space of nonnegative continuous functions on $\mathbb{R}^{d}$ with compact support) and $\langle\mu, \varphi\rangle$ denotes the integral $\int \varphi d \mu$. We also write $\|\mu\|=$ $\langle\mu, \mathbf{1}\rangle$ for the total mass of $\mu$.

If $\alpha=0$, then the number of families in $B$ (i.e., \#\{i: $\left.\chi_{i}(B)>0\right\}$ ) has a Poisson distribution with expectation $\mathbf{Q}(\chi: \chi(B)>0)$. If $\alpha(B)>0$, then a "continuum of families" contributes to $Y(B)$. This motivates the following definition.

DEFINITION 1.1. We say that the local biodiversity of the infinitely divisible random measure $Y$ is:

- finite if $\alpha=0$ and $\mathbf{Q}(\chi: \chi(B)>0)<\infty$ for every compact set $B$,

- countably infinite if $\alpha=0$ and $\mathbf{Q}(\chi: \chi(B)>0)=\infty$ for every open set $B \neq \varnothing$,

- uncountably infinite if $\alpha(B)>0$ for every open set $B \neq \varnothing$. 
Note that this distinction is exhaustive if the distribution of $Y$ is translation invariant.

As a trivial example, we mention $Y=\mathscr{f}_{t} \mu$, where $\mathscr{f}_{t}$ is the heat flow at time $t>0$ and $\mu \neq 0$ is a finite measure. In this case, obviously $Y$ has uncountably infinite local biodiversity.

As a second example, we consider in $d \geq 3$ the equilibrium super-Brownian motion $\left(\varrho_{t}\right)_{t \in \mathbb{R}}$. It is easily verified that $\varrho_{0}$ has finite local biodiversity. In fact, for general $Y$ to have finite local biodiversity it is sufficient and necessary that

$$
\mathbf{P}[Y(B)=0]>0 \quad \text { for any compact set } B \text {. }
$$

This follows from the simple observation that if $\alpha=0$,

$$
\mathbf{Q}(\chi: \chi(B)>0)=-\log \mathbf{P}[Y(B)=0] .
$$

Coming back to the equilibrium of super-Brownian motion, it is easily verified that for every compact set $B, \mathbb{P}_{-\infty, i_{c} l}\left[\varrho_{0}(B)=0\right]>0$.

The situation is quite different for CSBM and this is the content of our main result.

Theorem 1 (Biodiversity). Let $d=3$. For $\mathbb{P}_{-\infty, i_{c} l}$ almost all $\varrho$ the random measure $X_{0}^{\varrho}$ (under the distribution $P_{-\infty, i_{r} l}^{\varrho}$ ) has countably infinite local biodiversity.

The intuitive reasons for this behavior are as follows:

(i) In three dimensions the catalyst $\varrho$ lives on such a thin time-space set that small amounts of reactant mass can percolate to $B$ along catalystfree regions. In contrast, this is not possible for classical SBM: Here too small portions of mass (immigrating from outer space) get killed before they reach $B$.

(ii) The catalyst is not so thin that the reactant can sustain a deterministic component. Thus its genetic abundance is not as "rich" as that of the heat flow.

1.2. Instantaneous propagation of matter. The key ingredient for the proof of Theorem 1 is an instantaneous propagation of the reactant matter. Like the heat flow, the three-dimensional reactant spills out mass everywhere in space immediately. This property contrasts to the compact support property of classical SBM (see [13]) and, for example, one-dimensional CSBM where the (time-homogeneous) catalyst is a certain stable random measure (see [5]). An instantaneous propagation of matter for a super-Lévy process was first established by Perkins [15]; see also [9] for a generalization and a slimmer proof. 
Before we formulate our proposition, we introduce some notation that helps to define CSBM in a somewhat more general setup. Let $\mathscr{M}_{f}\left(\mathbb{R}^{d}\right)=\{\mu \in$ $\left.\mathscr{M}\left(\mathbb{R}^{d}\right):\|\mu\|<\infty\right\}$ and define the space of tempered measures $\mathscr{M}^{\prime}\left(\mathbb{R}^{d}\right)=$ $\cup_{p>d} \mathscr{M}_{p}\left(\mathbb{R}^{d}\right)$, where

$$
\mathscr{M}_{p}\left(\mathbb{R}^{d}\right)=\left\{\mu \in \mathscr{M}\left(\mathbb{R}^{d}\right):\left\langle\mu,\left(1+\|\bullet\|^{2}\right)^{-p / 2}\right\rangle<\infty\right\} .
$$

$\mathscr{M}^{\prime}\left(\mathbb{R}^{d}\right)$ is the state space for both $\varrho$ and $X^{\varrho}$. Denote by $\mathbb{P}_{t, \mu}$ the law of $\varrho$ started at time $t$ in the state $\mu \in \mathscr{M}^{\prime}\left(\mathbb{R}^{d}\right)$ and, for given $\varrho$, denote by $P_{t, m}^{\varrho}$ the law of $X^{\varrho}$ started at time $t$ in the state $m \in \mathscr{M}^{\prime}\left(\mathbb{R}^{d}\right)$. Let $\ll$ denote absolute continuity and $\approx$ denote equivalence of measures.

So that $X^{\varrho}$ can be defined properly, an additional restriction applies to $\mu$. The crucial property is that we can define the collision local time (see [10], Theorem 4.1) of a Brownian particle with $\varrho$ started in $\mu$ as a so-called nice branching functional. We call such a measure $\mu$ admissible. The class of admissible $\mu$ has not been characterized yet. However, $\mu$ is known to be admissible if, for example, it is " $\eta$-diffusive" in the sense of [11]. Here we mention only that the Lebesgue measure $l$, any $\mu \ll l$ with bounded density and $\mathbb{P}_{t, i_{c} l}$-almost all $\varrho_{0}$ are admissible, where $t \in[-\infty, 0)$ if $d=3$ and $t \in(-\infty, 0)$ if $d=2$. (This has been shown for in [11] only for $t \in(-\infty, 0)$, but follows easily for $d=3$ and $t=-\infty$. In fact, $\eta$-diffusivity is essentially a local property. However, for any compact $B \subset \mathbb{R}^{3}$, the total variation $\left\|\mathbb{P}_{t, i_{c} l}\left[\varrho_{0 \mid B} \in \bullet\right]-\mathbb{P}_{-\infty, i_{c} l}\left[\varrho_{0 \mid B} \in \bullet\right]\right\|_{\mathrm{TV}}$ tends to 0 as $t \rightarrow-\infty$, as can be seen, for example, by a simple cluster decomposition.)

Now we can formulate our proposition on the instantaneous propagation of the reactant matter.

Proposition 1.2 (Instantaneous propagation of matter). Assume $d=3$, that $\mu \in \mathscr{M}^{\prime}\left(\mathbb{R}^{d}\right)$ is admissible and that $m \in \mathscr{M}^{\prime}\left(\mathbb{R}^{d}\right), m \neq 0$. Then for all $t>0$,

$$
\mathbb{E}_{0, \mu}\left[P_{0, m}^{\varrho}\left[l \ll X_{t}^{\varrho} \mid X_{t}^{\varrho} \neq 0\right]\right]=1 .
$$

Together with the result of $[11]$ that says that $\mathbb{P}_{0, \mu}$ a.s. the reactant's states $X_{t}^{\varrho}$ are absolutely continuous w.r.t. $l$, we get the following corollary.

CoROllary 1.3. Assume $d=3$, that $\mu \in \mathscr{M}^{\prime}\left(\mathbb{R}^{d}\right)$ is admissible and that $m \in \mathscr{M}^{\prime}\left(\mathbb{R}^{d}\right), m \neq 0$. Then for all $t>0$,

$$
\mathbb{E}_{0, \mu}\left[P_{0, m}^{\varrho}\left[l \approx X_{t}^{\varrho} \mid X_{t}^{\varrho} \neq 0\right]\right]=1 .
$$

The reason Proposition 1.2 is true is that the catalyst is so thin in $d=3$ that it will not hit thin (time-space) cylinders connecting two points. Through those tubes, reactant mass propagates from one point to all other points in space immediately. It might seem reasonable to expect such a behaviour also for $d=2$. However, here the catalyst does hit the tubes (more formally, in $d=2$, lines are not polar for super-Brownian motion). To mimic an argument as for $d=3$, we would have to establish a percolation argument for the complement of the time-space support of two-dimensional super-Brownian motion. 
It would be nice to know whether $\left(\operatorname{supp}\left(\varrho_{t}\right)\right)^{c}$ (the complement of the support of $\varrho_{t}$ ) is connected in two dimensions. If it is connected, then we can essentially use the previously outlined argument to show an instantaneous propagation of reactant matter also in this case. One idea to show connectedness is to use an argument of dependent percolation for a discretization of the space. However, so far, efforts in this direction have failed.

On the other hand, if $\left(\operatorname{supp}\left(\varrho_{t}\right)\right)^{c}$ is not connected, then it might still be the case that the catalyst is so thin that the reactant can go through it without getting killed. In fact, the case studied in [5] is of this nature. There a situation is considered where in dimension 1 the time-homogeneous catalyst is supported by such a thin (though dense) set that the reactant propagates everywhere immediately.

1.3. Finite mass extinction. Another question we address in this paper is that of long-term extinction or finite time extinction of finite reactant masses in a catalyst started in Lebesgue measure. More precisely, assume that $\varrho_{0}=$ $i_{c} l, i_{c}>0$, and $X_{0}=m \in \mathscr{M}_{f}\left(\mathbb{R}^{d}\right)$. Is it true that $X_{t}^{\varrho} \rightarrow 0, \mathbb{E}_{0, i_{c} l}\left[P_{0, m}^{\varrho}\right]$-almost surely or even $\mathbb{E}_{0, i_{l} l}\left[P_{0, m}^{\varrho}\left[X_{t}^{\varrho}=0\right]\right] \rightarrow 1$ as $t \rightarrow \infty$ ?

The corresponding question for classical SBM is very simple to answer. Assume for the moment that the SBM $\varrho$ is started with a finite initial measure $\mu \in \mathscr{M}_{f}\left(\mathbb{R}^{d}\right)$. Then the total mass process $\left(\left\|\varrho_{t}\right\|\right)_{t \geq 0}$ is simply Feller's branching diffusion with initial mass $\|\mu\|$ this is the diffusion on $[0, \infty)$ with infinitesimal generator $\left.\sqrt{2 x}\left(\partial^{2} / \partial x^{2}\right)\right]$. Hence $\mathbb{P}_{0, \mu}\left[\varrho_{t}=0\right]=\exp (-\|\mu\| / t)$ and we have extinction in finite time:

$$
\lim _{t \rightarrow \infty} \mathbb{P}_{0, \mu}\left[\varrho_{t}=0\right]=1, \quad \mu \in \mathscr{M}_{f}\left(\mathbb{R}^{d}\right) .
$$

This is in contrast to the behavior of the reactant $X^{\varrho}$. In [1], Theorem 5, it was shown that if $d=1$, then for $\mathbb{P}_{0, i_{c} l}$ a.a. $\varrho$ under $P_{0, m}^{\varrho}$ the total mass process $\left(\left\|X_{t}^{\varrho}\right\|\right)_{t \geq 0}$ is an $L^{2}$-bounded martingale and hence converges almost surely to a random variable with full expectation $\|m\|$ (persistence) and finite variance.

However, for $d=2,3$ the reactant's behaviour is quite different. In the long run, the catalyst is not as scarce as in $d=1$ and so we do not have persistence of finite reactant mass, not even long-term survival. However, we do not have extinction in finite time (at least for $d=3$ ) either. Here is our result.

THEOREM 2 (Finite mass extinction). Let $d=3$ and $m \in \mathscr{M}_{f}\left(\mathbb{R}^{d}\right), m \neq 0$. Then there is no finite time extinction for the reactant

$$
\mathbb{E}_{0, i_{c} l}\left[P_{0, m}^{\varrho}\left[X_{t}^{\varrho} \neq 0\right]\right]=1, \quad t \geq 0 .
$$

However, for $d=2$ or $d=3$ there is extinction in the long-term limit:

$$
\mathbb{E}_{0, i_{c} l}\left[P_{0, m}^{\varrho}\left[\lim _{t \rightarrow \infty}\left\|X_{t}^{\varrho}\right\|=0\right]\right]=1 .
$$


The reason we do not have finite time extinction is simple to explain. The key is the instantaneous propagation of matter (Proposition 1.2). At time $t=0$, reactant mass is instantaneously spilled everywhere in space. For every $t>0$ and $\varepsilon \in(0, t)$ there are tubes $(x+B) \times[\varepsilon, t]$ in the complement of the timespace support of $\varrho$. In these tubes, the reactant can survive until time $t$ because it dominates heat flow with absorption at the boundary of $x+B$. We convert this idea into a rigorous proof in Section 4.1.

The die-out in (1.9) holds because the collision local time of a typical Brownian particle of the reactant moving in the translation invariant catalyst goes to $\infty$ as $t \rightarrow \infty$. Hence the typical particle undergoes branching for a total infinite amount of time, which leads to extinction (see Section 4.2). The situation is different in dimension $d=1$ : Here the collision local time remains bounded because the catalyst is very scarce (clusters are of order $t$ apart and have extensions of order $t^{1 / 2}$ only).

REMARK. Statement (1.8) depends on the assumption $d=3$ only by the instantaneous propagation of matter property (1.6). It would be true also for $d=2$ if we could show (1.6) also for $d=2$, which seems to be a reasonable statement.

1.4. Outline. The rest of the paper is organized as follows. In Section 2 we give the short proof of Proposition 1.2. In Section 3 we prove Theorem 1. It takes some technical effort involving exit measures to turn the reasoning following Theorem 2 into a rigorous proof. This is the content of Section 4.

2. Instantaneous propagation of matter. Here we prove Proposition 1.2. The rough idea is that any two time-space points $(t, x)$ and $(t, y)$ can be connected by a straight line that is not hit by $\operatorname{supp}(\varrho)$. [We denote by $\operatorname{supp}(\varrho) \subset$ $[0, \infty) \times \mathbb{R}^{d}$ the closed support of the measure $d t \varrho_{t}(d x)$.] Hence also a time-space neighbourhood of this line is catalyst-free. If there is reactant matter around $y$ at time $t$, then a small amount has percolated according to the heat flow through the "tube" to $x$. If we condition on $X_{t}^{\varrho} \neq 0$, then there is some $y$ such that there is reactant matter around $(t, y)$. Thus there is some matter around $(t, x)$ also and we are done. The following lines make this idea rigorous.

For $x, y \in \mathbb{R}^{d}$ define $\overline{x y}=\{\alpha x+(1-\alpha) y: \alpha \in[0,1]\}$. It is well known that lines are polar for three-dimensional super-Brownian motion (see [8], Theorem 3.5(ii)). More precisely, if we denote by $\operatorname{supp}(\varrho)$ the time-space support of $\varrho$, then for $t>0$,

$$
\mathbb{P}_{0, i_{c} l}[\operatorname{supp}(\varrho) \cap(\{t\} \times \overline{x y})=\varnothing]=1 .
$$

For $t>0$ fixed, $x, y \in \mathbb{R}^{d}$ and $\varepsilon \in(0, t)$ define the tube

$$
T(t, x, y, \varepsilon)=(t-\varepsilon, t+\varepsilon) \times \bigcup_{z \in \overline{x y}} B(z, \varepsilon),
$$


where $B(x, R)$ is the open ball with radius $R$ centered at $x$. From (2.1) we get

$$
\lim _{\varepsilon \rightarrow 0} \mathbb{P}_{0, i_{c} l}\left[\int_{T(t, x, y, \varepsilon)} d s \varrho_{s}(d z)=0\right]=1 .
$$

[Note that both (2.1) and (2.3) fail if $d \leq 2$; see, again, [8], Theorem 3.5(ii).] Thus with probability 1 , for any $x, y \in \mathbf{Q}^{3}$ (the three-dimensional rational numbers) there exists a random number $\varepsilon(x, y)>0$ such that $\int_{T(t, x, y, \varepsilon(x, y))} d s \varrho_{s}(d z)=0$.

We know that $X_{t}^{\varrho}$ is absolutely continuous with respect to Lebesgue measure and that its density function $\xi_{t}^{\varrho}$ is continuous off $\operatorname{supp}(\varrho)$ and solves the heat equation (see [11]). Let $S_{t}=\left\{z \in \mathbb{R}^{3}:(t, z) \in \operatorname{supp}(\varrho)\right\}$. Assume now that $X_{t}^{\varrho} \neq 0$. Whereas $l\left(S_{t}\right)=0$ a.s., it suffices to show for $x \in S_{t}^{\mathrm{c}}:=\mathbb{R}^{3} \backslash S_{t}$ that $\xi_{t}^{\varrho}(x)>0$. By the continuity of the density we need to show this only for all $x \in \mathbf{Q}^{\mathbf{3}} \cap \mathbf{S}_{\mathbf{t}}^{\mathbf{c}}$. Note that $X_{t}^{\varrho} \neq 0$ implies that there exists some $y \in \mathbf{Q}^{\mathbf{3}} \cap \mathbf{S}_{\mathbf{t}}^{\mathrm{c}}$ with $\xi_{t}^{\varrho}(y)>0$. Because $T(t, x, y, \varepsilon(x, y)) \rightarrow[0, \infty),(s, z) \mapsto \xi_{s}^{\varrho}(z)$ solves the heat equation, we have in fact $\xi_{s}^{\underline{o}}(z)>0$ for all $(s, z) \in T(t, x, y, \varepsilon(x, y))$.

3. Infinite biodiversity. In this section we prove Theorem 1 . The statement we have to show consists of two parts: (i) the deterministic component of $X_{0}^{\varrho}$ vanishes and (ii) the canonical measure of $\{\chi: \chi(B)>0\}$ is infinite for every open set $B \neq \varnothing$.

3.1. Vanishing deterministic component. We start with the proof of the first statement. Recall that we consider the bivariate process $\left(\varrho_{t}, X_{t}^{\varrho}\right)_{t \in \mathbb{R}}$ in the equilibrium. That is, $\varrho$ is sampled according to $\mathbb{P}_{-\infty, i_{c} l}$ and for given $\varrho$ the law of $X^{\varrho}$ is $P_{-\infty, i_{r} l}^{\varrho}$. For convenience we agree that for fixed $\varrho$, we define all random variables related to superprocesses in the catalytic medium $\varrho$ on the same sufficiently large underlying probability space whose law we denote by $P^{\varrho}$. For the deterministic and random component of a random measure $Y$ over this probability space we simply write $\operatorname{det}^{\varrho} Y:=\operatorname{ess} \inf Y$ and $\operatorname{ran}^{\varrho} Y:=$ $Y-\operatorname{det}^{\varrho} Y$. Note that for any measurable set $B \subset \mathbb{R}^{d}$,

$$
\left(\operatorname{det}^{\varrho} Y\right)(B) \leq \operatorname{det}^{\varrho}(Y(B))=-\lim _{\lambda \rightarrow \infty} \lambda^{-1} \log E^{\varrho}\left[e^{-\lambda Y(B)}\right],
$$

where both sides may equal $\infty$. Strict inequality holds, for example, if $V$ is a nontrivial random variable in $\mathbb{R}^{d}, Y=\delta_{V}$ and $B=\mathbb{R}^{d}$. However, there is equality if $Y$ is infinitely divisible. In fact, if we denote by $\mathbf{Q}$ the canonical measure of $Y$ and let $\alpha$ be as in (1.3), then $\alpha=\operatorname{det}^{\varrho} Y$ and the right-hand side of (3.1) equals

$$
\operatorname{det}^{\varrho}(Y(B))=\alpha(B)+\lim _{\lambda \rightarrow \infty} \int_{\mathscr{M}\left(\mathbb{R}^{d}\right)} \mathbf{Q}(d \chi) \lambda^{-1}\left(1-e^{-\lambda \chi(B)}\right)=\alpha(B),
$$

where the second equality comes from the fact that the integral is finite for every $\lambda$ and the integrand decreases to 0 as $\lambda$ approaches $\infty$.

Now we can state that the deterministic component of $X_{0}^{\varrho}$ vanishes. 
Lemma 3.1. Let $d=3$. For $\mathbb{P}_{-\infty, i_{c} l}$ almost all $\varrho$,

$$
\operatorname{det}^{\varrho} X_{0}^{\varrho}=0 .
$$

PROOF. It suffices to show that the expected deterministic component $\mathbb{E}_{-\infty, i_{c} l}\left[\operatorname{det}^{\varrho} X_{0}^{\varrho}\right]$ disappears. Assume the contrary. Then, by the spatial shiftinvariance of $\mathbb{P}_{-\infty, i_{c} l}$, we have

$$
\mathbb{E}_{-\infty, i_{c} l}\left[\operatorname{det}^{\varrho} X_{0}^{\varrho}\right]=b l \quad \text { for some } b \in\left(0, i_{r}\right] .
$$

Fix a sample $\varrho$ such that $\operatorname{det}^{\varrho} X_{0}^{\varrho} \neq 0$. Given $X_{0}^{\varrho}$, introduce independent CSBM $X^{\varrho, d}=\left(X_{t}^{\varrho, d}\right)_{t \geq 0}$ and $X^{\varrho, r}=\left(X_{t}^{\varrho, r}\right)_{t \geq 0}$ (in the medium $\varrho$ ) with initial states $X_{0}^{\varrho, d}=\operatorname{det}^{\varrho} X_{0}^{\varrho}$ and $X_{0}^{\varrho, r}=\operatorname{ran}^{\varrho} X_{0}^{\varrho}$. By the branching property we may assume that

$$
X_{1}^{\varrho}=X_{1}^{\varrho, d}+X_{1}^{\varrho, r} .
$$

We claim that

$$
\operatorname{det}^{\varrho} X_{1}^{\varrho, r}=0 .
$$

In fact, fix a compact $A \subset \mathbb{R}^{3}$ and an $\varepsilon>0$. For $R>0$, the fixed $\varrho$ and given $X_{0}^{\varrho, r}$, as before we decompose $X^{\varrho, r}$ into independent catalytic super-Brownian motions $X^{\varrho, r, i}, i=1,2$, with catalyst $\varrho$ and initial states

$$
X_{0}^{\varrho, r, 1}=X_{0}^{\varrho, r} \mathbf{1}_{B(0, R)}, \quad X_{0}^{\varrho, r, 2}=X_{0}^{\varrho, r} \mathbf{1}_{B(0, R)^{c}} .
$$

[Recall that $B(x, R)$ is the open ball with radius $R$ centered at $x \in \mathbb{R}^{d}$.] Assume that

$$
X_{1}^{\varrho, r}=X_{1}^{\varrho, r, 1}+X_{1}^{\varrho, r, 2} .
$$

Note that also $\operatorname{det}^{\varrho} X_{1}^{\varrho, r}=\operatorname{det}^{\varrho} X_{1}^{\varrho, r, 1}+\operatorname{det}^{\varrho} X_{1}^{\varrho, r, 2}$. By the Markov property and the expectation formula (recall that $\mathscr{f}_{t}$ is the heat flow),

$$
E^{\varrho}\left[X_{1}^{\varrho, r, 2}(A)\right]=E^{\varrho}\left[\left\langle\mathscr{S}_{1}\left(X_{0}^{\varrho, r} \mathbf{1}_{B(0, R)^{c}}\right), \mathbf{1}_{A}\right\rangle\right]=E^{\varrho}\left[\left\langle X_{0}^{\varrho, r} \mathbf{1}_{B(0, R)^{c}}, \mathscr{\rho}_{1} \mathbf{1}_{A}\right\rangle\right] .
$$

Hence we can choose $R$ large enough such that

$$
E^{\varrho}\left[X_{1}^{\varrho, r, 2}(A)\right] \leq \varepsilon
$$

(recall that $X_{1}^{\varrho, r, 2} \leq X_{1}^{\varrho, r} \leq X_{1}^{\varrho}$, where the latter term has $P^{\varrho}$ expectation $\left.i_{r} l\right)$.

Whereas $X_{0}^{\varrho, r}$ has a vanishing deterministic component, its infinite divisibility implies that also $\left\|X_{0}^{\varrho, r, 1}\right\|=X_{0}^{\varrho, r}(B(0, R))$ does not have a deterministic component [recall (3.2)]. Thus for all $\delta>0$ we have $P^{\varrho}\left[\left\|X_{0}^{\varrho, r, 1}\right\|<\delta / 2\right]>0$. Noting that $\left(\left\|X_{t}^{\varrho, r, 1}\right\|\right)_{t \geq 0}$ is a martingale, we get

$$
P^{\varrho}\left[\left\|X_{1}^{\varrho, r, 1}\right\| \leq 2\left\|X_{0}^{\varrho, r, 1}\right\| \mid\left\|X_{0}^{\varrho, r, 1}\right\|\right] \geq \frac{1}{2} \quad \text { almost surely, }
$$


and thus

$$
P^{\varrho}\left[\left\|X_{1}^{\varrho, r, 1}\right\|<\delta\right] \geq \frac{1}{2} P^{\varrho}\left[\left\|X_{0}^{\varrho, r, 1}\right\|<\delta / 2\right]>0 .
$$

Hence $\operatorname{det}^{\varrho} X_{1}^{\varrho, r, 1}=0$. Combining this with (3.7), the claim (3.5) follows.

Recall that we fixed $\varrho$ such that $X_{0}^{\varrho, d}=\operatorname{det}^{\varrho} X_{0}^{\varrho} \neq 0$. This implies $\operatorname{ran}^{\varrho} \times$ $X_{1}^{\varrho, d} \neq 0$ with positive $P^{\varrho}$ probability; thus, $E^{\varrho}\left[\operatorname{ran}^{\varrho} X_{1}^{\varrho, d}\right] \neq 0$. By translation invariance of $\mathbb{P}_{-\infty, i_{c} l}$ there exists an $a>0$ such that

$$
\mathbb{E}_{-\infty, i_{c} l}\left[E^{\varrho}\left[\operatorname{ran}^{\varrho} X_{1}^{\varrho, d}\right]\right]=a l .
$$

Finally, by the decomposition (3.4) and by (3.5), $\operatorname{det}^{\varrho} X_{1}^{\varrho}=\operatorname{det}^{\varrho} X_{1}^{\varrho, d}=$ $X_{1}^{\varrho, d}-\operatorname{ran}^{\varrho} X_{1}^{\varrho, d}$ and, therefore, we can build the annealed expectation to obtain

$$
\begin{aligned}
b l & =\mathbb{E}_{-\infty, i_{c} l}\left[E^{\varrho}\left[\operatorname{det}^{\varrho} X_{1}^{\varrho}\right]\right] \\
& =\mathbb{E}_{-\infty, i_{c} l}\left[E^{\varrho}\left[X_{1}^{\varrho, d}\right]\right]-\mathbb{E}_{-\infty, i_{c} l}\left[E^{\varrho}\left[\operatorname{ran}^{\varrho} X_{1}^{\varrho, d}\right]\right]=(b-a) l .
\end{aligned}
$$

This is clearly a contradiction and completes the proof.

3.2. The equilibrium reactant charges every set. We complete the proof of Theorem 1 by showing that the reactant's canonical measure is infinite on $\{\chi: \chi(B)>0\}$ for any open set $B \neq \varnothing$. Recall from (1.5) that it is enough to show that the reactant charges any open set:

$$
\mathbb{E}_{-\infty, i_{c} l}\left[P_{-\infty, i_{r} l}^{\varrho}\left[X_{0}^{\varrho}(B)=0\right]\right]=0 \quad \text { for any open } B \neq \varnothing .
$$

However, this follows from the instantaneous propagation of matter (Proposition 1.2), and Theorem 1 is now completely proved.

4. Finite mass extinction. In this section we prove Theorem 2. The proofs of the two statements (no finite time extinction, but long-term extinction) are methodologically different and we present them in separate subsections.

4.1. No finite time extinction. Recall that here $\varrho$ is distributed according to $\mathbb{P}_{0, i_{c} l}$ and $X^{\varrho}$ is distributed according to $P_{0, m}^{\varrho}$ for some nonvanishing $m \in$ $\mathscr{M}_{f}\left(\mathbb{R}^{d}\right)$. To show that there is no finite time extinction, we rely again on the instantaneous propagation of reactant matter (Proposition 1.2). Additionally, we need the following property of the $\operatorname{support} \operatorname{supp}(\varrho) \subset[0, \infty) \times \mathbb{R}^{d}$ of the the measure $d t \varrho_{t}(d x)$. Recall that $B(x, r) \subset \mathbb{R}^{d}$ is the open ball of radius $r$ centered at $x$.

LEMMA 4.1 (Empty tubes in the catalyst). Assume $d \geq 2$. For every $t>0$, $\varepsilon \in(0, t)$ and $\mathbb{P}_{0, i_{c} l}$ a.a. $\varrho$ there exists a $z \in \mathbb{Z}^{d}$ such that $\operatorname{supp}(\varrho) \cap([\varepsilon, t] \times$ $B(z, 1))=\varnothing$. 
Proof. Fix $t>\varepsilon>0$. For $z \in \mathbb{R}^{d}$ define the event

$$
A(z)=\{\operatorname{supp}(\varrho) \cap([\varepsilon, t] \times B(z, 1))=\varnothing\} .
$$

Fix $R>0$ and note that by the branching property of SBM we have

$$
\mathbb{P}_{0, i_{c} l}[A(z)]=\mathbb{P}_{0, i_{c} l 1_{B(z, R)}}[A(z)] \cdot \mathbb{P}_{0, i_{c} l 1_{B(z, R)}}[A(z)] .
$$

Obviously,

$$
\mathbb{P}_{0, i_{c} l 1_{B(z, R)}}[A(z)] \geq \mathbb{P}_{0, i_{c} l 1_{B(z, R)}}\left[\varrho_{\varepsilon}=0\right]>0, \quad R>0 .
$$

For the other factor in (4.1) we need an estimate on the range of SBM (see [4], Theorem 3.3a): Fix $R>2 t^{1 / 2}$. Then there exists a $c>0$ such that for $x \in \mathbb{R}^{d}$,

$$
f_{z}(x):=\mathbb{P}_{0, \delta_{x}}\left[A(z)^{\mathrm{c}}\right] \leq c \exp \left(-\|x-z\|^{2} / 2 t\right) .
$$

Noting that $\log (1-s)>-2 s$ for $s \in\left[0, \frac{1}{2}\right]$ we see that for $R$ sufficiently large,

$$
\begin{aligned}
\mathbb{P}_{0, i_{c} l \mathbf{1}_{B(z, R)^{c}}}[A(z)] & =\exp \left(i_{c} \int_{B(z, R)^{c}} \log \left(1-f_{z}(x)\right) d x\right) \\
& \geq \exp \left(-2 c i_{c} \int_{B(z, R)^{c}} \exp \left(-\|x-z\|^{2} / 2 t\right) d x\right)>0 .
\end{aligned}
$$

Thus $\mathbb{P}_{0, i_{c} l}[A(z)]>0$ and we can infer from the ergodic theorem (note that $\mathbb{P}_{0, i_{c} l}$ is spatially ergodic) $\mathbb{P}_{0, i_{c} l}\left[\cup_{z \in \mathbb{Z}^{d}} A(z)\right]=1$.

With this lemma we are almost done. Recall that we specify on $d=3$. Fix $\delta \in(0,1)$ and $\varepsilon \in(0, t)$ such that $\mathbb{P}_{0, i_{c} l}\left[P_{0, m}^{\varrho}\left[X_{\varepsilon}^{\varrho} \neq 0\right]>1-\delta\right]>1-\delta$. Now choose such a $\varrho$ and an $X_{\varepsilon}^{\varrho}$ that are in the described event. We may assume that $\varrho \in \bigcup_{z \in \mathbb{Z}^{3}} A(z)$. Let $z \in \mathbb{Z}^{3}$ such that $\varrho \in A(z)$. By Proposition 1.2 we have $X_{\varepsilon}^{\varrho}\left(B\left(z, \frac{1}{2}\right)\right)>0$. Denote by $\left(\mathscr{\rho}_{s}^{z}\right)_{s \geq 0}$ the semigroup of heat flow with absorption at $\mathbb{R}^{3} \backslash B(z, 1)$. Then we can estimate

$$
X_{t}^{\varrho}(B(z, 1)) \geq\left\|\mathscr{S}_{t-\varepsilon}^{z} X_{\varepsilon}^{\varrho}\right\| \geq\left\|\mathscr{S}_{t-\varepsilon}^{z}\left(\mathbf{1}_{B(z, 1 / 2)} X_{\varepsilon}^{\varrho}\right)\right\|>0 .
$$

Hence

$$
\mathbb{P}_{0, i_{c} l}\left[P_{0, m}^{\varrho}\left[X_{t}^{\varrho} \neq 0\right]>1-\delta\right]>1-\delta .
$$

Now let $\delta \rightarrow 0$. This shows the first assertion of Theorem 2 .

4.2. Long-term extinction. In this subsection we show the second statement of Theorem 2. We first outline the idea of the proof. An "infinitesimal particle" of $X^{\varrho}$ performs (independently of $\varrho$ ) Brownian motion $W$ on $\mathbb{R}^{d}$ whose law and expectation are denoted by $\mathbf{P}_{x}$ and $\mathbf{E}_{x}$, respectively, $x \in \mathbb{R}^{d}$. The branching along such a reactant's path $W$ is governed by the collision local time $L_{[W, \varrho]}$ of $W$ with the catalyst $\varrho$. This can be defined for $d \leq 3$ as the $L^{2}$ limit (see [1] and [10])

$$
L_{[W, \varrho]}(0, t)=\lim _{\varepsilon \rightarrow 0} \int_{0}^{t} d s \int_{\mathbb{R}^{d}} \varrho_{s}(d z) p_{\varepsilon}\left(z, W_{s}\right), \quad t \geq 0,
$$


where $\left(p_{t}\right)_{t>0}$ is the family of standard Brownian transition densities. [For $d \geq 4, \operatorname{supp}(\varrho)$ is polar for $W$; that is, $W$ does not collide with $\varrho$, and $X^{\varrho}$ degenerates to the heat flow.]

Loosely speaking, the idea is that by a fixed large time $T$, most infinitesimal reactant particles have collected a large amount of collision local time, say at least $K$. With a high probability (namely the extinction probability of Feller's branching diffusion at time $K$ ), all these particles have died. The expected number of particles that have collected less collision local time is $\mathbf{E}_{0}\left[\mathbb{P}_{0, i_{c} l}\left[L_{[W, \varrho]}(0, T)<K\right]\right]$ which tends to 0 as $T \rightarrow \infty$.

Infinite total collision local time. However intuitively appealing and verbally simple to describe the idea is, we need some technical effort to make it rigorous. We start by showing that the collision local time $L_{[W, \varrho]}$ increases in fact to $\infty$ almost surely if $d=2,3$. Note that this contrasts with dimension $d=1$, where $L_{[W, \varrho]}(0, \infty)<\infty$ almost surely (see [1], Proposition 8 ). The difference between dimensions 1 and 2 is that $\varrho$ dies out locally almost surely if $d=1$, whereas it does so only in probability if $d=2$. In the latter case, the clusters recur to visit the window of observation at arbitrarily late times. Of course, for $d=3$ there is no extinction and a law of large numbers applies.

Proposition 4.2. Let $d=2,3$. Then

$$
\mathbf{E}_{x}\left[\mathbb{P}_{0, i_{c} l}\left[L_{[W, \varrho]}(0, \infty)=\infty\right]\right]=1, \quad x \in \mathbb{R}^{d} .
$$

PRoOF. By spatial homogeneity of Brownian motion and the law of $\varrho$, it suffices to consider $x=0$. For $d=3$, the claim follows from a law of large numbers (see [2]):

$$
\mathbf{E}_{0}\left[\mathbb{P}_{0, i_{c} l}\left[\lim _{t \rightarrow \infty} t^{-1} L_{[W, \varrho]}(0, t)=i_{c}\right]\right]=1 .
$$

For $d=2$, there is no law of large numbers. Rather $L_{[W, \varrho]}$ is self-similar in the sense that (see [2])

$$
\begin{aligned}
\mathbf{E}_{0} & {\left[\mathbb{P}_{0, i_{c} l}\left[L_{[W, \varrho]}(0, t) \in \bullet\right]\right] } \\
& =\mathbf{E}_{0}\left[\mathbb{P}_{0, i_{c} l}\left[T^{-1} L_{[W, \varrho]}(0, T t) \in \bullet\right]\right], \quad t, T>0 .
\end{aligned}
$$

Note that the events $\left\{L_{[W, \varrho]}(0, \infty)>T\right\}$ are decreasing in $T>0$ and due to the self-similarity all have the same $\mathbb{E}_{0} \mathbb{P}_{0, i_{c} l}$ probability. Hence

$$
\begin{aligned}
\mathbf{E}_{0}\left[\mathbb{P}_{0, i_{c} l}\left[L_{[W, \varrho]}(0, \infty)=\infty\right]\right] & =\mathbf{E}_{0}\left[\mathbb{P}_{0, i_{c} l}\left[\bigcap_{T>0} L_{[W, \varrho]}(0, \infty)>T\right]\right] \\
& =\mathbf{E}_{0}\left[\mathbb{P}_{0, i_{c} l}\left[\bigcup_{T>0} L_{[W, \varrho]}(0, \infty)>T\right]\right] \\
& =\mathbf{E}_{0}\left[\mathbb{P}_{0, i_{c} l}\left[L_{[W, \varrho]}(0, \infty)>0\right]\right] .
\end{aligned}
$$


Analogously

$$
\begin{aligned}
\left.\mathbf{E}_{0}\left[\mathbb{P}_{0, i_{c} l} l L_{[W, \varrho]}(0, \infty)>0\right]\right] & =\mathbf{E}_{0}\left[\mathbb{P}_{0, i_{c} l}\left[\bigcup_{t \geq 1} L_{[W, \varrho]}(0, t)>0\right]\right] \\
& =\mathbf{E}_{0}\left[\mathbb{P}_{0, i_{c} l}\left[L_{[W, \varrho]}(0,1)>0\right]\right]>0,
\end{aligned}
$$

where the inequality follows from $\mathbf{E}_{0}\left[\mathbb{E}_{0, i_{c} l}\left[L_{[W, \varrho]}(0,1)\right]\right]=i_{c}>0$. Using the same trick for $\left\{L_{[W, \varrho]}(t, \infty)=0\right\}$, we get

$$
\begin{aligned}
\mathbf{E}_{0}\left[\mathbb{P}_{0, i_{c} l}\left[L_{[W, \varrho]}(0, \infty)=0\right]\right] & =\mathbf{E}_{0}\left[\mathbb{P}_{0, i_{c} l}\left[\bigcap_{t>0}\left\{L_{[W, \varrho]}(t, \infty)=0\right\}\right]\right] \\
& =\mathbf{E}_{0}\left[\mathbb{P}_{0, i_{c} l}\left[\bigcup_{t>0}\left\{L_{[W, \varrho]}(t, \infty)=0\right\}\right]\right] \\
& =\mathbf{E}_{0}\left[\mathbb{P}_{0, i_{c} l}[\varrho \in A(W)]\right],
\end{aligned}
$$

where

$$
A(W)=\bigcup_{t>0}\left\{\varrho: L_{[W, \varrho]}(t, \infty)=0\right\} .
$$

We are done if we can show a suitable 0-1 law for the last expression in the equation array (4.12), which would imply that the expressions in (4.11) and (4.10) all equal 1. This is a spinoff of the subsequent lemma, which then completes the proof of the proposition.

Lemma 4.3 (0-1 law). Assume $d \leq 3$. Then one of the following two alternatives holds:

$$
\mathbf{P}_{x}\left[W: \mathbb{P}_{0, i_{c} l}[\varrho \in A(W)]=0\right]=1, \quad x \in \mathbb{R}^{d}
$$

or

$$
\mathbf{P}_{x}\left[W: \mathbb{P}_{0, i_{c} l}[\varrho \in A(W)]=1\right]=1, \quad x \in \mathbb{R}^{d} .
$$

Proof. Again by spatial homogeneity, either alternative holds if it does for $x=0$.

We first show that

$$
\mathbf{P}_{0}\left[\mathbb{P}_{0, i_{c} l}[\varrho \in A(W)] \in\{0,1\}\right]=1 .
$$

In fact, let $\varrho=\sum_{z \in \mathbb{Z}^{d}} \varrho^{z}$ be a decomposition of $\varrho$ into independent SBM starting in $\varrho_{0}^{z}=l 1_{z+[0,1)^{d}}$. Whereas $\mathbb{P}_{0, i_{c} l}\left[\sum_{z \in Z} \varrho_{t}^{z}=0\right] \rightarrow 1$ as $t \rightarrow \infty$, for any finite $Z \subset \mathbb{Z}^{d}$, the event $A(W)$ is in the completion of the tail field:

$$
A(W) \in \bigcap_{n \in \mathbb{N}} \sigma\left(\varrho^{z},\|z\| \geq n\right) \quad\left(\bmod \mathbb{P}_{0, i_{c} l}\right) .
$$

The tail field is $\mathbb{P}_{0, i_{c} l}$ trivial and (4.16) follows. 
Assume now that (4.14) does not hold. Hence $\mathbf{P}_{0}\left[W: \mathbb{P}_{0, i_{c} l}[\varrho \in A(W)]>\right.$ $0]>0$ and by (4.16),

$$
\mathbf{P}_{0}\left[W: \mathbb{P}_{0, i_{c} l}[\varrho \in A(W)]=1\right]>0 .
$$

Fix $t>0$. Note that $A(W)=\left\{\varrho: \varrho_{t+\bullet} \in A\left(W_{t+\bullet}\right)\right\}$; hence, we have

$$
\begin{aligned}
\mathbb{P}_{0, i_{c} l}[\varrho \in A(W)] & =\mathbb{P}_{0, i_{c}}\left[\varrho_{t+\bullet} \in A\left(W_{t+\bullet}\right)\right] \\
& =\mathbb{P}_{0, i_{c} l}\left[\varrho_{t+\bullet} \in A\left(W_{t+\bullet}-W_{t}\right)\right],
\end{aligned}
$$

where in the last step we used the spatial translation invariance of $\mathbb{P}_{0, i_{c} l}$. Hence, $W \mapsto \mathbb{P}_{0, i_{l} l}[\varrho \in A(W)]$ is measurable w.r.t. the tail field of the increments of $W$ and thus constant. By (4.18) we get (4.15).

REMARK 4.4. Note that the proof of Proposition 4.2 shows for $d=2$ even the stronger statement that for all $x \in \mathbb{R}^{2}$ and $\mathbf{E}_{x} \mathbb{P}_{0, i_{c} l}$ almost surely,

$$
L_{[W, \varrho]}(0, t)>0 \quad \text { and } \quad L_{[W, \varrho]}(t, \infty)=\infty, \quad t>0 .
$$

Exit measures. Now we make precise the idea of the collision local time collected by individual "infinitesimal particles" from the introduction of this subsection. Note that the idea of using exit measures for this purpose was employed successfully also by Dawson, Fleischmann and Mueller [3] (see also [12]).

Choose a sample $\varrho$ according to $\mathbb{P}_{0, i_{c} l}$. Recall that $d=2,3$. From Proposition 4.2 it follows that the following stopping times of $W$ are $\mathbf{P}_{x}$ almost surely finite:

$$
\tau_{K}=\inf \left\{t>0: L_{[W, \varrho]}(0, t) \geq K\right\}, \quad K \geq 0 .
$$

For each of these stopping times we could define Dynkin's stopped measure, which is approximately what we want. However, this is a (random) measure on the particles' path space and needs a construction of historical CSBM. This is not too hard to do, but we prefer to follow a slightly more elementary route.

We would like to consider the joint process of $W$ and its collision local time $L_{[W, \varrho]}$. It will, however, be convenient to introduce the trivariate (timehomogeneous) Markov process $\widetilde{W}=(W, L, I)$ on $\mathbb{R}^{d} \times[0, \infty) \times[0, \infty)$, where for $t \geq 0$,

$$
\begin{aligned}
I_{t} & =t+I_{0}, \\
L_{t} & =L_{0}+L_{[W, \varrho]}\left(I_{0}, I_{t}\right),
\end{aligned}
$$

with $I_{0}, L_{0} \geq 0$. For this enriched process $\widetilde{W}$, started in $\left(W_{0}, 0,0\right)$, each $\tau_{K}$ is an exit time,

$$
\tau_{K}=\inf \left\{t>0: \tilde{W}_{t} \notin A\right\}
$$

where

$$
A=\mathbb{R}^{d} \times[0, K) \times[0, \infty) .
$$


We can define the catalytic branching process $\tilde{X}^{\varrho}$, which is the catalytic superprocess (on $\mathbb{R}^{d} \times[0, \infty) \times[0, \infty)$ ) with underlying motion $\widetilde{W}$, with "critical binary" branching and with branching functional $L$. For an initial state $\tilde{m} \in \mathscr{M}_{f}\left(\mathbb{R}^{d} \times[0, \infty) \times[0, \infty)\right)$, we denote its law by $P_{\tilde{m}}^{\varrho}$. Note that for

$$
\tilde{m} \text { concentrated on } \mathbb{R}^{d} \times\{0\} \times\{0\} \text { with }
$$

$$
\tilde{m}(\bullet \times[0, \infty) \times[0, \infty))=m(\bullet),
$$

$X_{T}^{\varrho}(\bullet)$ and $\tilde{X}_{T}^{\varrho}(\bullet \times[0, \infty) \times\{T\})$ coincide in distribution. Hence we are done if we can show that

$$
\limsup _{T \rightarrow \infty} P_{\widetilde{m}}^{\varrho}\left[\left\|\tilde{X}_{T}^{\varrho}\right\|>\varepsilon\right]=0, \quad \varepsilon>0 .
$$

[Note that the $P_{0, m}^{\varrho}$ almost sure convergence statement (1.9) of Theorem 2 follows from this because $\left(\left\|X_{t}^{\varrho}\right\|\right)_{t \geq 0}$ is a nonnegative martingale and hence almost surely convergent.]

For an exit time $\tau$ of a domain $A \subset \mathbb{R}^{d} \times[0, \infty) \times[0, \infty)$, we can define the exit measure $\tilde{X}_{\tau}^{\varrho}$ by the following procedure. Let $\tilde{X}^{\varrho, \tau}$ be defined as $\tilde{X}^{\varrho}$ but with $\widetilde{W}_{\tau \wedge \text { • }}$ instead of $\widetilde{W}$ as motion (and with $L_{\tau \wedge \bullet}$ as branching functional). That is, the infinitesimal particles get frozen when they reach the boundary $\partial A$ of $A$. Finally, let $\widetilde{X}_{\tau}^{o}$ be the measure that is supported by $\partial A$ and that is obtained as the monotone limit

$$
\tilde{X}_{\tau}^{\varrho}=\lim _{t \rightarrow \infty} \tilde{X}_{t}^{\varrho, \tau}(\partial A \cap \bullet) .
$$

Note that for $\tilde{m}$ as in (4.24) and $\tau$ a finite exit time we have

$$
E_{\tilde{m}}^{\varrho}\left[\tilde{X}_{\tau}^{\varrho}\right]=\int m(d x) \mathbf{P}_{x}\left[\tilde{W}_{\tau} \in \bullet \cdot\right] .
$$

For the exit measures $\tilde{X}_{\tau}^{\varrho}$ we have the so-called special Markov property, which amounts to saying that if $\tau \leq \sigma$ are exit times of $\widetilde{W}$, then we obtain $\widetilde{X}_{\sigma}^{\varrho}$ from $\widetilde{X}_{\tau}^{\varrho}$ by starting the process afresh (cf. [6], Theorem 1.6 and [7], Theorem 1.5). More precisely, if for $\varphi \in C_{c}^{+}\left(\mathbb{R}^{d} \times[0, \infty) \times[0, \infty)\right)$ and $x \in \mathbb{R}^{d} \times[0, \infty) \times[0, \infty)$, we define

$$
\left(V_{\sigma}^{\varrho} \varphi\right)(x)=-\log E_{\delta_{x}}^{\varrho}\left[\exp \left(-\left\langle\tilde{X}_{\sigma}^{\varrho}, \varphi\right\rangle\right)\right]
$$

then

$$
E_{\tilde{m}}^{\varrho}\left[\exp \left(-\left\langle\tilde{X}_{\sigma}^{\varrho}, \varphi\right\rangle\right)\right]=E_{\tilde{m}}^{\varrho}\left[\exp \left(-\left\langle\tilde{X}_{\tau}^{\varrho}, V_{\sigma}^{\varrho} \varphi\right\rangle\right)\right] .
$$

In particular, if $\partial A=A^{1} \cup A^{2}, A^{1} \cap A^{2}=\varnothing$ and $\tilde{X}^{\varrho, 1}$ and $\tilde{X}^{\varrho, 2}$ are independent processes (given their initial states) of the type introduced already with $\widetilde{X}_{0}^{\varrho, i}=$ $\widetilde{X}_{\tau}^{\varrho}\left(A^{i} \cap \bullet\right), i=1,2$, then $\tilde{X}_{\sigma}^{\varrho}$ is equal in distribution to their sum,

$$
\tilde{X}_{\sigma}^{\varrho} \stackrel{\mathrm{d}}{=} \tilde{X}_{\sigma}^{\varrho, 1}+\tilde{X}_{\sigma}^{\varrho, 2} .
$$

Now we come back to our concrete situation. Here $\sigma \equiv T, \tau=\tau_{K} \wedge T$, $A=\mathbb{R}^{d} \times[0, K) \times[0, T)$, and $\partial A=A^{1} \cup A^{2}$, where $A^{1}=\mathbb{R}^{d} \times[0, K) \times\{T\}$, 
and $A^{2}=\mathbb{R}^{d} \times\{K\} \times[0, T]$. Assume that $\tilde{m}$ is as in (4.24). Hence, using (4.27) we get

$$
\begin{aligned}
E^{\varrho}\left[\left\|\tilde{X}_{T}^{\rho, 1}\right\|\right] & =E_{\tilde{m}}^{\varrho}\left[\tilde{X}_{\tau_{K} \wedge T}^{\varrho}\left(A^{1}\right)\right] \\
& =\int m(d x) \mathbf{P}_{x}\left[\tau_{K}>T\right] \rightarrow 0, \quad T \rightarrow \infty .
\end{aligned}
$$

On the other hand, $\tilde{X}_{\tau_{K} \wedge T}^{\varrho}\left(A^{2}\right)=\tilde{X}_{\tau_{K}}^{\varrho}\left(A^{2}\right)$, thus

$$
P^{\varrho}\left[\tilde{X}_{T}^{\varrho, 2} \neq 0\right] \leq P_{\tilde{m}}^{\varrho}\left[\tilde{X}_{\tau_{K}}^{\varrho}\left(A^{2}\right)>0\right] .
$$

Now we employ a result of [3], Lemma 14, that the process $\left(\left\|\tilde{X}_{\tau_{t}}^{o}\right\|\right)_{t \geq 0}$ is Feller's branching diffusion. Hence we have $P_{\widetilde{m}}^{\varrho}\left[\left\|\tilde{X}_{\tau_{K}}^{\varrho}\right\|>0\right]=1-e^{-\|m\| / K}$ and thus

$$
P^{\varrho}\left[\left\|\widetilde{X}_{T}^{\varrho, 2}\right\|>0\right] \leq 1-e^{-\|m\| / K} .
$$

Combining (4.30), (4.31) and (4.33), where we first let $T \rightarrow \infty$ and then $K \rightarrow$ $\infty$, we get that (4.25) holds.

Acknowledgments. This work was accomplished partly while the second author was a guest at the Weierstrass Institute for Applied Analysis and Stochastics in Berlin. It is a pleasure to thank the WIAS for hospitality and support. We would like to thank also Ed Perkins and an anonymous referee for valuable comments.

\section{REFERENCES}

[1] Dawson, D. A. and Fleischmann, K. (1997). A continuous super-Brownian motion in a super-Brownian medium. J. Theoret. Probab. 10 213-276.

[2] Dawson, D. A. and Fleischmann, K. (1997). Longtime behavior of a branching process controlled by branching catalysts. Stochastic Process. Appl. 71 241-257.

[3] Dawson, D. A., Fleischmann, K. and Mueller, C. (2000). Finite time extinction of superprocesses with catalysts. Ann. Probab. 28 000-000.

[4] Dawson, D. A., Iscoe, I. and Perkins, E. A. (1989). Super-Brownian motion: Path properties and hitting probabilities. Probab. Theory Related Fields 83 135-205.

[5] Dawson, D. A., LI, Y. and Mueller, C. (1995). The support of measure-valued branching processes in a random environment. Ann. Probab. 23 1692-1718.

[6] Dynkin, E. B. (1991). Branching particle systems and superprocesses. Ann. Probab. 19 1157-1194.

[7] Dynkin, E. B. (1991). Path processes and historical superprocesses. Probab. Theory Related Fields 90 1-36.

[8] Dynkin, E. B. (1992). Superdiffusions and parabolic nonlinear differential equations. Ann. Probab. 20 942-962.

[9] Evans, S. N. and Perkins, E. A. (1991). Absolute continuity results for superprocesses with some applications. Trans. Amer. Math. Soc. 325 661-681.

[10] Evans, S. N. and Perkins, E. A. (1994). Measure-valued branching diffusions with singular interactions. Canad. J. Math. 46 120-168.

[11] Fleischmann, K. and Klenke, A. (1999). Smooth density field of catalytic super-Brownian motion. Ann. Appl. Probab. 9 298-318.

[12] Fleischmann, K. and Mueller, C. (1997). A super-Brownian motion with a locally infinite catalytic mass. Probab. Theory Related Fields 107 325-357. 
[13] IsCOE, I. (1988). On the supports of measure-valued critical branching Brownian motion. Ann. Probab. 16 200-221.

[14] Klenke, A. (2000). A review on spatial catalytic branching. In Stochastic Models. A Conference in Honour of Professor Don Dawson (L. Gorostiza and G. Ivanoff, eds.) 245-263. Amer. Math. Soc., Providence, RI.

[15] Perkins, E. (1990). Polar sets and multiple points for super-Brownian motion. Ann. Probab. $18453-491$.

WEIERSTRASS-INSTITUT FÜR

ANGEWANDTE ANALYSIS UND STOCHASTIK

MOHRENSTRASSE 39

10117 BERLIN

GERMANY

E-MAIL: fleischm@wias-berlin.de
Mathematisches InstituT

UNIVERSITÄT ERLANGEN-NÜRNBERG

BISMARCKSTRASSE $1 \frac{1}{2}$

91054 ERLANGEN

GERMANY

E-MAIL: klenke@mi.uni-erlangen.de 